\title{
A review of current analytical methods for the determination of prebiotics in foods
}

\author{
Tran Hung Son ${ }^{1,2}$, Vu Thi Thanh $A n^{1}$, Mac Thi Thanh Hoa ${ }^{1}$ \\ Cao Cong Khanh ${ }^{1}$, Le Thi Hong Hao ${ }^{1}$ \\ ${ }^{1}$ National Institute for Food Control, Hanoi, Vietnam
}

${ }^{2}$ Natural Product Informatics Research Center, Korea Institute of Science and Technology (KIST),

Gangwon, Republic of Korea

(Received: 03/05/2021; Accepted: 24/06/2021)

\section{Abstract}

Prebiotics are a group that improves the human intestinal microbiota. The relationship between prebiotics and human health has been an area of increasing interest in recent years. Fructo-oligosaccharides and galacto-oligosaccharides are the two important groups of prebiotics with beneficial effects on human health. Besides that, more and more carbohydrates have shown a good potential to be prebiotics has been studied. This study presents a review of the characteristics, properties, and analytical methods used for the determination of prebiotics in food products. Most of the methods found using chromatographic methods, especially HPAEC - PAD but also another method, such as spectrometric methods have been presented. A discussion was presented highlighting the drawbacks of current analytical methods and the need to develop these methods to analyze the complex food samples containing prebiotics.

Keywords: prebiotics, review, foods, analytical methods, fructo-oligosaccharides, galactooligosaccharides.

\section{INTRODUCTION}

Prebiotics are known as non-digestible carbohydrates selectively fermented to stimulate microbiota in the human intestinal. In 1995, prebiotics was firstly defined by Gibson and Roberfroid as "a non-digestible food ingredient that beneficially affects the host by selectively stimulating the growth and/or activity of one or a limited number of bacteria in the colon, and thus improves host health" [1]. In 2008, the $6^{\text {th }}$ Meeting of the International Scientific Association of Probiotics and Prebiotics (ISAPP) defined "dietary prebiotics" as "a selectively fermented ingredient that results in specific changes in the composition and/or activity of the gastrointestinal microbiota, thus conferring benefit(s) upon host health" [2].

There are some criteria used to classify a compound as a prebiotic. Firstly, it should be resistant to acidic $\mathrm{pH}$ of stomach, cannot be hydrolysed by mammalian enzymes, and also should not be absorbed in the gastrointestinal tract. Secondly, it can be fermented by intestinal microbiota, and finally, the growth and/or activity of the intestinal bacteria can be selectively stimulated by this compound and this process improves the host's health [2]. Some prebiotics can be extracted from plant sources, but most are synthesized commercially using enzymatic or chemical methods. Overall, prebiotics are manufactured by four major routes: direct extraction,

${ }^{\star}$ Corresponding author: Tel 0389811395

Email:tranhungson113@gmail.com 
controlled hydrolysis, trans-glycosylation, and chemical process [3].

Most of the prebiotics are carbohydrates and two criteria can be applied to distinguish fiber from carbohydrate-derived prebiotics. Firstly, fibers are carbohydrates with a degree of polymerization (DP) equal or higher than three. Secondly, endogenous enzymes in the small intestine cannot hydrolyse them. It should be taken into account that fiber solubility or fermentability is not crucial. The most common prebiotics are inulin, fructo-oligosaccharides/ oligofructose (FOS), $\beta$-glucan, galacto-oligosaccharides (GOS), however, other complex saccharides, and fibers have been recently considered as prebiotics [4].

Prebiotics has a beneficial effect on human health, and has greater potential for supplement into a broader range of common food products as they have both technical and nutritional properties. They act as food sources for the growth of probiotic bacteria (bifidobacteria and lactic acid bacteria), thus enhance the gastrointestinal functions and immune system. Prebiotics also increase the absorption of calcium and magnesium, reduce blood glucose, and lipid levels. [5].

Nowadays, food products that contain prebiotics are becoming more and more popular and diverse. Therefore, the application of methods to analyse the content of prebiotics in the above products to evaluate product quality is very important and necessary. Due to the importance of prebiotics in food products, this paper presents a review of the classification of prebiotics, their properties and the most important - the analytical methods and techniques used for the determination of prebiotics in foods. Among them, spectrophotometric and chromatographic methods are most commonly used in the quantification of prebiotics.

\section{TYPES OF PREBIOTICS}

\subsection{Fructans}

Fructans consist of inulin and fructooligosaccharide or oligofructose. Their structure is a linear chain of fructose with $\beta-(2 \rightarrow 1)$ linkage (Figure 1). Inulin has a degree of polymerization (DP) of up to 60, while the DP of FOS is often less than ten. Inulin and FOS can be easily dissolved in hot water but very little in cold water and alcohol. Inulin and FOS are generally found in plants, bacteria, and some fungi [6].

\subsection{Galacto-oligosaccharides}

Galacto-oligosaccharides (GOS), the product of lactose extension, are classified into two subgroups: The GOS with excess galactose at C3, C4, or C6 and the GOS are manufactured from lactose through transglycosylation activity. The $\beta$-galactosidase enzymes are used to complete the transglycosylation reaction. The end product of this reaction is mainly a mixture of trito pentasaccharides with galactose in $\beta(1 \rightarrow 6), \beta(1 \rightarrow 3)$, and $\beta(1 \rightarrow 4)$ linkages (Figure 2 ). This type of GOS is also termed as trans-Galacto-oligosaccharides (TOS) [7].

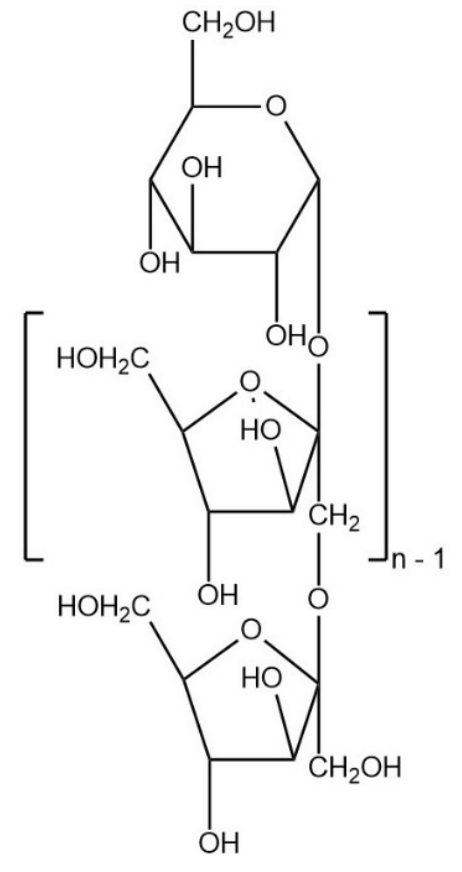

Inulin $(n=2-60)$

Fruto-oligosaccharides $(n=2-10)$

Figure 1. Structure of fructooligosaccharides/inulin 


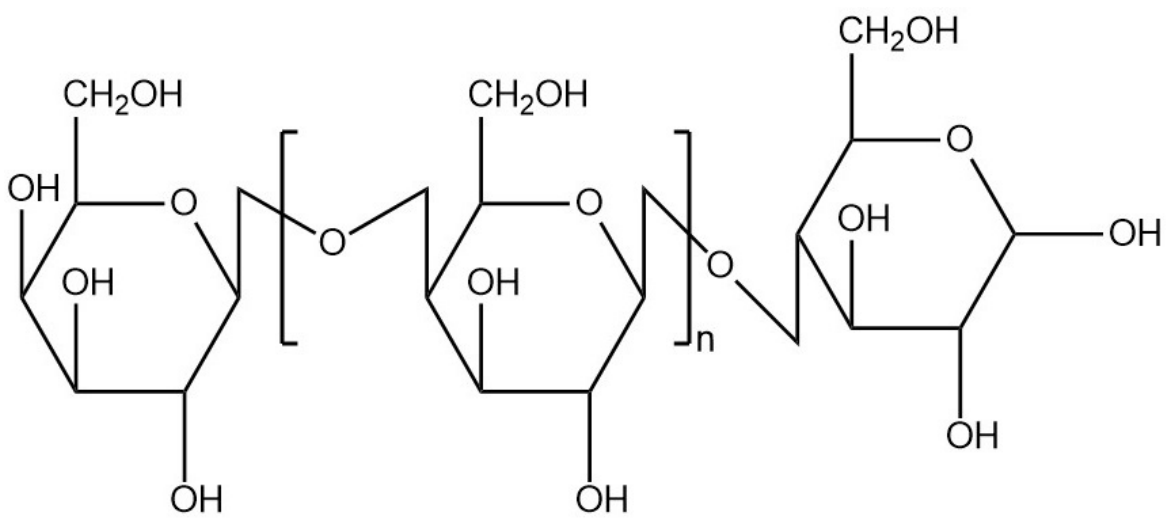

Figure 2. The structure of trans-Galacto-oligosaccharides

\subsection{Polydextrose}

Polydextrose is a polysaccharide composed of randomly cross-linked glucose units with various types of glycosidic bonding, with $\alpha-(1 \rightarrow 6)$ bonds are predominate (Figure 3$)$. Polydextrose has an average degree of polymerization of 12 and an average molecular weight of approximately 2,000 . Polydextrose is produced through a polymerization of glucose, using sorbitol and citric acid as the catalyst. Therefore, the polydextrose ingredient contains about $10 \%$ of sorbitol and $1 \%$ of citric acid. Because of the complexity of the molecule, human digestive enzymes are unable to hydrolyze the molecule [8].

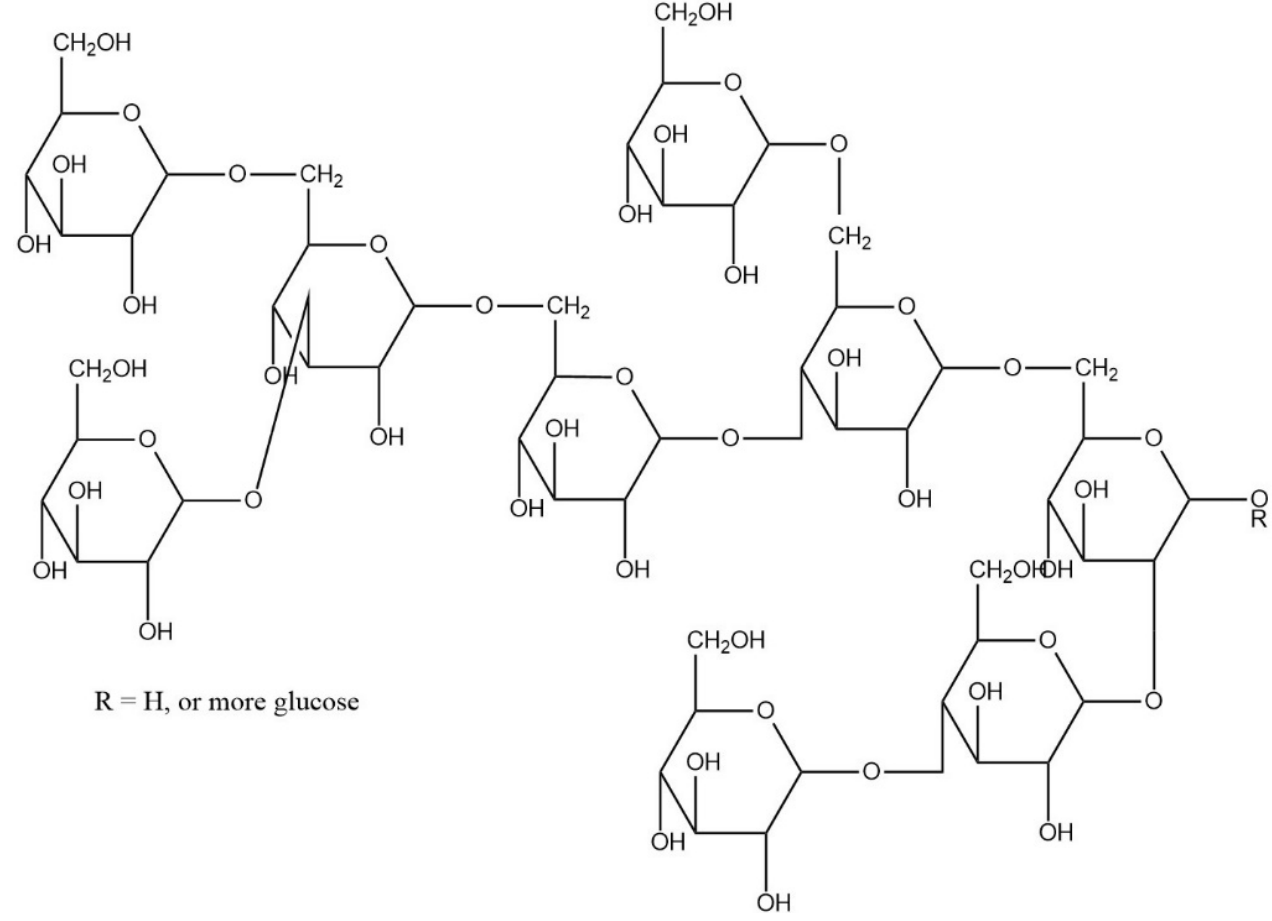

Figure 3. The structure of polydextrose

\section{4. $\beta$-glucan}

$\beta$-Glucan is a water-soluble polysaccharide that consists of glucose units. $\beta$-glucans form a natural component of the cell walls of bacteria, fungi, yeast, and cereals such as oat and barley. 
The most common forms of $\beta$-glucans are those comprising D-glucose units with $\beta$-1,3 links. Yeast and fungal $\beta$-glucans contain 1-6 side branches, while cereal $\beta$-glucans contain both $\beta$-1,3 and $\beta-1,4$ backbone bonds (Figure 4) [9].
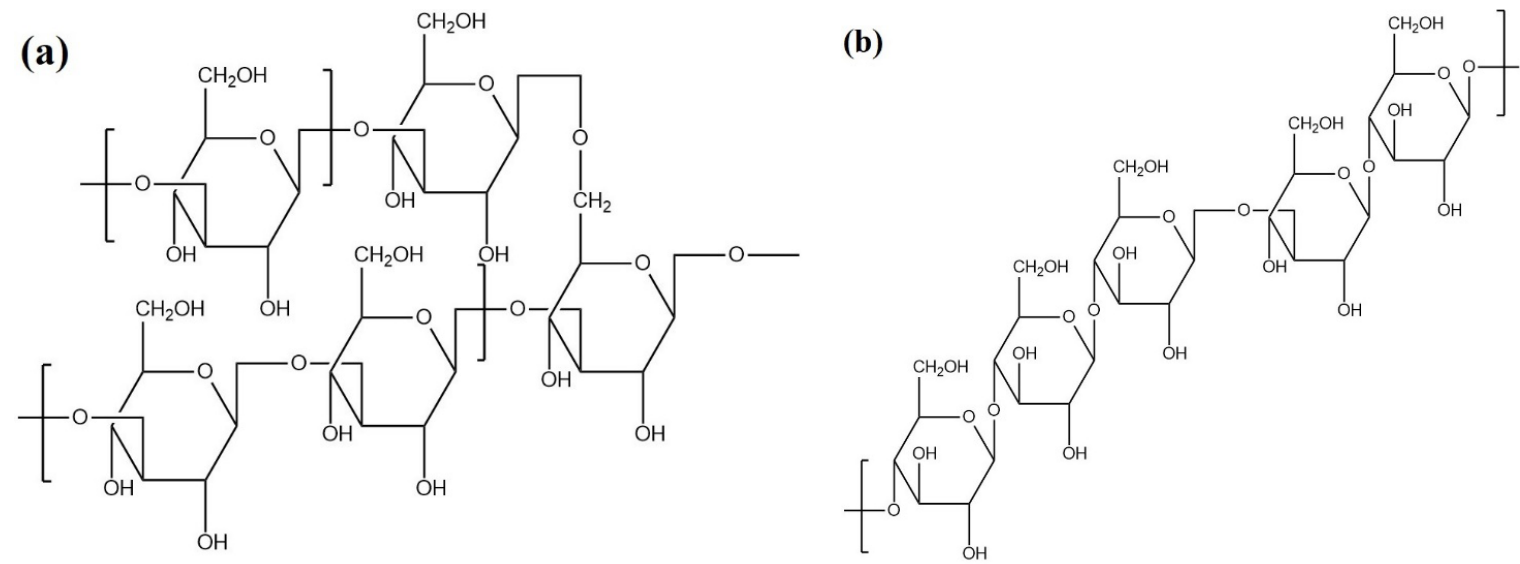

Figure 4. The structure of a) $\beta-(1-3,1-6)$-glucan and b) $\beta-(1-3,1-4)$-glucan

\subsection{Other Prebiotics}

In addition to prebiotics that have been widely used such as FOS, GOS, and polydextrose, several other polysaccharides or oligo-saccharides have also shown great potential for use as a prebiotic in food. These include other substances such as isomalto-oligosaccharides (IMO), xylo-oligosaccharides (XOS), soybean oligosaccharides, lactosucrose, etc. [3].

\section{EFFECTS OF PREBIOTICS ON HEALTH AND THEIRS APPLICATIONS IN FOODS}

\subsection{Effects of prebiotics on human health}

One of the most important effects of prebiotics is their impact on the human gut microbiota. As an energy source for gut microorganisms, prebiotics can alter the composition and function of these microorganisms. These effects of prebiotics were observed in subjects of all ages, from infants to the elderly. In the composition of breast milk, Human Milk Oligosaccharides (HMOs) are a natural source of prebiotics that are extremely important for the health of the infant. For cow's milk-fed infants, a supplement at $8 \mathrm{~g} / \mathrm{L}$ milk concentration of a prebiotic blend with a ratio of 9 GOS: 1 FOS can simulate many of the physiological effects of HMOs, which is very low in cow's milk (100 times lower than in breast milk) [10]. In adults, the composition of the gut microbiota becomes much more complex with more than 500 different species of microorganisms. Diets containing prebiotics such as GOS or Inulin showed growth of beneficial microbial populations of Bifidobacterium and showed a decrease in harmful microorganisms such as Clostridia, Bacteriodes spp. and Enterobacteriaceae. For the elderly, studies show a significant decrease in beneficial bacteria such as Bifidobacterium, and an increase in harmful bacteria such as Clostridia. Diets containing prebiotics show potential in restoring populations of Bifidobacterium, and simultaneously reducing levels of harmful bacteria in the gut microbiota [11].

There are also studies on the impact of prebiotics on diseases related to the intestinal system. Irritable bowel syndrome (IBS) is a gastrointestinal syndrome characterized by chronic abdominal pain and altered bowel habits without any organic cause. Meanwhile, Crohn's Disease is a type of chronic, relapsing inflammatory bowel disease (IBD). A recent study showed that 
IBS symptoms improved after consuming $3.5 \mathrm{~g}$ of GOS/day for 12 weeks [12]. Another study reported that supplementation with $15 \mathrm{~g}$ FOS/day for three weeks increased the number of Bifidobacteria in the stool and improved Crohn's disease [13]. Prebiotics fermentation products, such as butyrate, may have a protective effect against colorectal cancer risk. Prebiotics also show potential in preventing intestinal infections. In vitro experiments have shown that GOS and lactulose can interfere with the adhesion of enteropathogenic bacteria E. coli [3].

In addition, recent studies have also shown the positive effects of prebiotics on human health such as improving the function of the immune system through increasing the growth of protective microorganisms, enhances the absorption of minerals such as calcium and magnesium. Moreover, prebiotics also have a positive effect on the body's metabolism, such as reducing blood triglyceride levels in patients with hyperlipidemia or using as a diet with low energy, low Glycemic Index (GI) for diabetics due to their digestive resistance to enzymes in the human digestive system [5]. Figure 5 summaries the effects of prebiotics on human health.

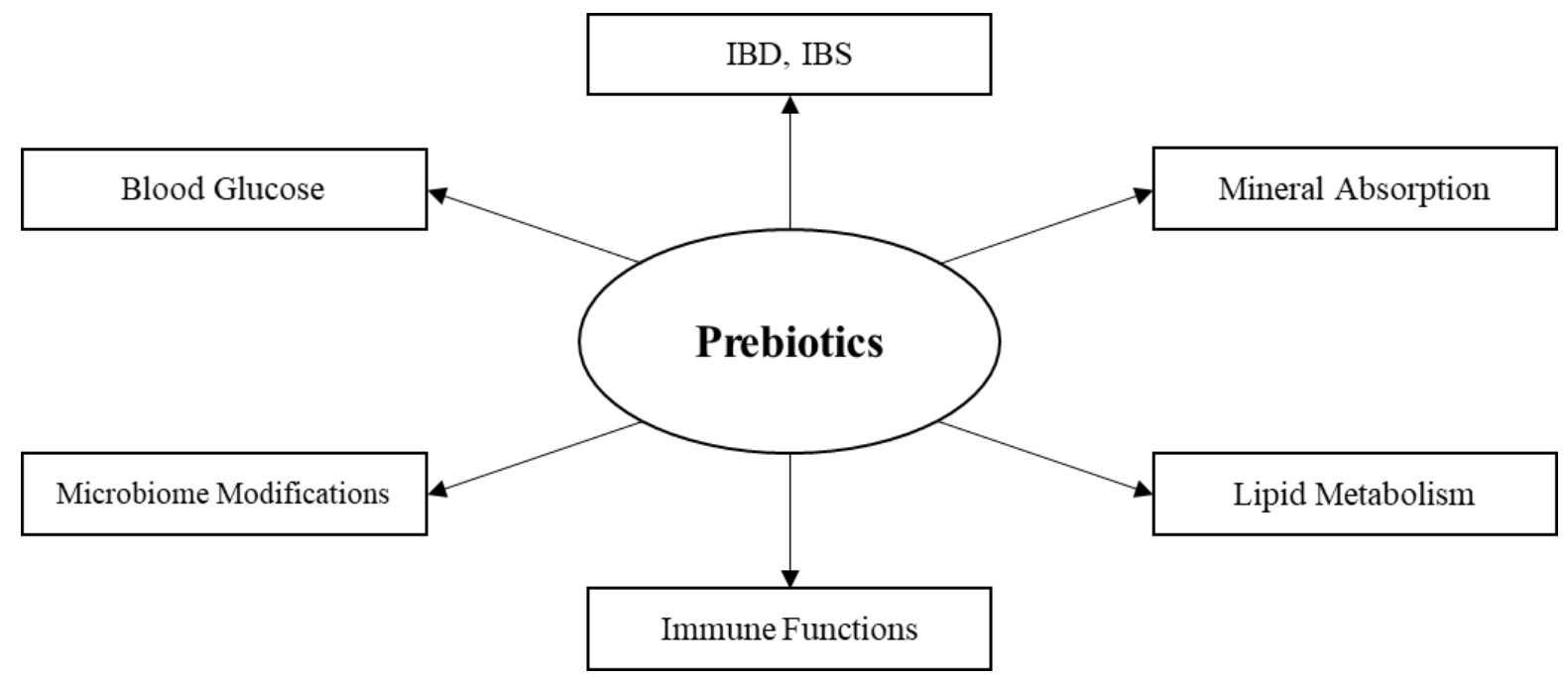

Figure 5. Beneficial effects of prebiotics on human health

\subsection{Application of prebiotics in foods}

The application of prebiotic should maintain the chemical stability of prebiotics to processing treatments of food, such as high temperature, low $\mathrm{pH}$, and Maillard reaction conditions. Therefore, if the prebiotic was degraded to its component monosaccharides and disaccharides or chemically altered, a prebiotic would no longer provide selective stimulation of beneficial microorganisms and it was unavailable for bacterial metabolism [14]. In fact, prebiotics is used in different foods such as in infant foods, fermented milk, cheese, cakes, biscuits, chocolates, sugar confectionery, soups, and sauces and the global demand has grown significantly over the previous ten years to amount to approximately 500,000 tons per year [15].

Currently, more and more prebiotics are used in different food products as a component that helps in the growth of health-promoting colon microorganisms particularly probiotics and offers additional health benefits. Many commercial dietary supplements are also added with oligosaccharide prebiotics. Concerning their addition to infant food, the European Commission's Scientific Committee on Food concluded in 2003 that the addition of oligosaccharides to infant formulas had no major worry and including the studied infant formulas (formulas adapted 
especially for 6- to 12 -month-old infants), up to a total concentration of $0.8 \mathrm{~g} / \mathrm{dL}$ in ready-tofeed formula products [16]. A study showed the effect of the addition of oligosaccharides having a concentration of $1 \mathrm{~g} / \mathrm{dL}$ to newly born infant formula for one month having $90 \%$ of GOSs and $10 \%$ of FOSs [17]. Another study demonstrated the effect of FOS supplemented infant formula for a 12-week study period. It showed that the infant growth was maintained without having any adverse effects [18]. Therefore, the supplement of prebiotics to infant food products showed to have a bifidogenic effect. Infant food formulas containing either GOS or FOS are now popularly marketed in Vietnam and all over the world.

\section{ANALYTICAL METHODS}

\subsection{Analytical techniques}

The review of the papers showed that some analytical techniques have been applied for the determination of prebiotics, namely, spectrometry, chromatography, capillary electrophoresis, and mass spectrometry method. However, the most common method used for the determination of prebiotic is high-performance anion exchange chromatography with pulsed amperometric detector (HPAEC-PAD). Table 1 introduces the reference methods for the determination of prebiotics.

Table 1. Approved methods for prebiotics

\begin{tabular}{ccc}
\hline Methods & Prebiotics & Analytical techniques \\
\hline AOAC 995.16 & B-Glucan & Enzymatic/Spectrophotometric methods \\
AOAC 997.08 & Fructans & Anion Exchange Chromatographic \\
AOAC 999.03 & Fructans & Method (HPAEC-PAD) \\
AOAC 2000.11 & Polydextrose & Anion Exchange Chromatographic \\
AOAC 2001.02 & trans-GOS & Method (HPAEC-PAD) \\
AOAC 2002.02 & Resistant starch & Anion Exchange Chromatographic \\
AOAC 2016.14 & Fructans & Method (HPAEC-PAD) \\
& & Anion Exchange Chromatographic \\
\hline
\end{tabular}

\subsubsection{Spectrometric methods}

Spectrometric methods are commonly used in determining the concentration of prebiotics in food samples. The general principle of these methods is the use of chemical agents such as acids, bases, or the use of specific enzymes to hydrolyze prebiotics in the form of polysaccharides or oligo-saccharides into their monomers - which are monosaccharides. Prebiotics content is calculated through the determination of monosaccharides content. There are two ways to determine the content of monosaccharides. The first way is to react the monosaccharides with chromogenic reagents, then the resulting color product is determined optically utilizing an ultraviolet-visible spectrometer at the appropriate wavelength. Second, monosaccharides 
are quantified indirectly using specific enzymes that catalyze a chemical reaction between the monosaccharides and another substance (usually NADPH), which the product forms (NADP) cause a variation in the absorbance at a specific wavelength.

Several methods belong to the group of spectrometric methods approved by the AOAC and AACC for quantification, such as fructans, $\beta$-glucan, resistant starch, or lactulose. AOAC Method 999.03 integrated enzyme treatments with spectrophotometric determination for the measurement of fructan and fructo-oligosaccharides. Test samples are extracted into hot water with $\mathrm{pH}$ maintained above 5.5. Extracts are incubated with a solution of sucrase/amylase, followed by a reduction with sodium borohydride to change the free monosaccharides to sugar alcohol. The mixtures are then incubated with fructanase for hydrolyzing fructan to fructose and glucose, followed by the addition of $p$-hydroxybenzoic acid hydrazide (PAHBAH) reagent and the absorbance is measured at $410 \mathrm{~nm}$ against a reagent blank. Total fructan content is calculated from the concentration of fructose in the hydrolysate [19].

AOAC Method 995.16 described a method for the quantification of $\beta$-glucans with a mixed structure (1-3,1-4)- $\beta$-D-glucan. The test samples are extracted in a buffer with $\mathrm{pH}$ 6.5. The extracts were then incubated with a mixture of lichenase and $\beta$-glucosidase enzymes to completely hydrolyze $\beta$-glucan to $\beta$-D-glucose. The amount of glucose formed is carried out to create an oxidation-reduction reaction with a mixture of glucose oxidase/peroxidase reagents. The absorbance of the formed color product was measured at $510 \mathrm{~nm}$ and $\beta$-glucan was calculated from the amount of glucose formed after the hydrolysis reaction [20].

AOAC Method 2002.02 illustrated a method for the quantification of resistant starch. Samples are incubated with a mixture of enzyme pancreatic $\alpha$-amylase/amyloglucosidase (AMG) for $16 \mathrm{~h}$ at $37^{\circ} \mathrm{C}$, during which time non-resistant starch is solubilized and hydrolyzed to $\mathrm{D}$-glucose by the combined action of the two enzymes. The reaction is terminated by the addition of ethanol or industrial methylated spirits (IMS, denatured ethanol), and the resistant starch is recovered as a pellet on centrifugation. Resistant starch in the pellet is then dissolved in $2 \mathrm{M} \mathrm{KOH}$. This solution is neutralized with acetate buffer, and the starch is quantitatively hydrolyzed to glucose with AMG. D-Glucose is measured with glucose oxidase/peroxidase reagent and this is a measure of the resistant starch content of the sample [21].

In general, spectrometric methods have the advantage of not requiring complex analytical equipment. Most laboratories can be equipped with a UV-VIS spectrophotometer to analyze the content of prebiotics. Furthermore, the determination of monosaccharides formed by enzymatic reactions has a very good specificity, which is sometimes not possible by chromatographic or electrochemical methods if the separation between monosaccharides causes a problem (in the case of glucose and galactose). However, the main disadvantage of spectrometric methods is the high limit of quantification (LOQ). This disadvantage leads to the difficulty in the analysis of the samples containing a low level of prebiotics.

\subsubsection{Chromatographic methods}

Chromatographic methods are most commonly used for the quantification of prebiotics. The general principle of the methods is similar to that of the spectrometric methods, that is the use of chemical agents such as acids and bases or the use of specific enzymes to hydrolyze the prebiotics in the form of polysaccharides or oligo-saccharides into monosaccharides molecules. Prebiotics content is calculated through the determination of monosaccharides content. 
Chromatographic methods generally provide a lower LOQ than spectrometric methods. However, the key issue in the use of chromatographic methods is the selection of stationary phase and mobile phase to ensure the separation of the monosaccharides in the mixture, and the selection of detectors to detect monosaccharides.

For stationary phase selection, both reverse phase and cation exchange chromatography have been the most common HPLC modes utilized for carbohydrate analysis till the suggestion of using HPAEC for this aim. Aminoalkyl-modified silica gel columns provide a good resolution; however, their stability is low and can be easily degraded. Many researchers have used amino columns to analyze fructo-oligosaccharides (FOS) of different DP using acetonitrile: water $(75: 25)$ as mobile phase, however, the resolution is not as good as that obtained for mono- and disaccharides and solubility problems appear [22-26]. Several stationary phases with highly polar sorbents such as cyano, hydroxyl, diol, etc. have been also used for carbohydrate analysis [27]. Analysis on these columns has in common that retention increases with the hydrophilicity of the stationary phase and the analytes and with decreasing hydrophilicity of solvents from the mobile phase. All of them are therefore grouped under the acronym HILIC (hydrophilic interaction chromatography). Currently, the most common type of stationary phase used is the anion exchange stationary phase. The appearance of HPAEC for carbohydrate analysis strongly improved knowledge about oligosaccharide composition of a wide variety of products [28]. Carbohydrates are negatively charged at high $\mathrm{pH}(\mathrm{pH}>13)$, and oligo- and polysaccharides up to DP50 can be separated by anion-exchange chromatography using $\mathrm{NaOH}$ as the mobile phase. A gradient of increasing concentration of sodium acetate is normally used to help elution of oligosaccharides.

Detector selection is also an important factor, determining the sensitivity of the method. Refractive index (RI) detectors are the most common detectors used for carbohydrate analysis although a lack of sensitivity is normally associated with them [29]. UV detectors at low wavelengths (below $210 \mathrm{~nm}$ ) show similar sensitivity to RI detectors however, they allow changes in temperature and gradient elution. Evaporative scattering detector (ELSD) is another choice for detection. This detector is universal, more sensitive than RI, and is compatible with elution gradients [29-32].

Pulsed Amperometric Detectors (PAD) are commonly coupled to HPAEC and allow the detection of non-derivatized carbohydrates at very low picomole levels. This detection provides high selectivity, only compounds oxidizable at the selected voltages being detected. PAD is composed of a working electrode of $\mathrm{Au}$ or $\mathrm{Pt}$, a stainless steel auxiliary electrode, and a reference electrode of $\mathrm{Ag} / \mathrm{AgCl}$ or $\mathrm{H}_{2}$. The Au electrode can catalyze the oxidation reactions and is the best choice for the detection of carbohydrates. Carbohydrates are detected by measuring the electrical current generated by their oxidation at the surface of the working electrode at the selected potential (E1). Next, the voltage is increased (E2) to oxidize the gold detector which causes desorption of the carbohydrate oxidation products. Finally, the potential is lowered (E3; negative potential) to reduce the electrode surface for the next pulse. The three potentials are applied for fixed times. Up to now, the use of the HPAEC-PAD method has shown the best performance in the analysis of prebiotics [28].

AOAC Method 997.08 was the first method approved by AOAC for the determination of fructans and their oligomers using the HPAEC-PAD method. Test samples are extracted with boiling water; the extract is hydrolyzed sequentially with amyloglucosidase and inulinase. Free 
fructose, glucose, and sucrose are separated and quantified by HPAEC-PAD in the extract before hydrolysis, then glucose and fructose after each of the two enzyme hydrolysis steps. Fructan content in the test sample is calculated by difference from the amount of each sugar in different solutions [33].

AOAC Method 2016.14 is the improvement of AOAC Method 997.03 for the determination of fructans and fructo-oligosaccharides. Test samples are extracted into hot water and the extracts are then incubated with a solution of sucrase, followed by a reduction with sodium borohydride to change the free monosaccharides to sugar alcohol. The mixtures are then incubated with fructanase for hydrolyzing fructan to fructose and glucose. Free fructose and glucose are separated and quantified by HPAEC-PAD. Fructan content in the test sample was calculated by the amount of fructose and glucose in the mixture [34].

Trans-Galacto-oligosaccharides (TGOS) are manufactured oligosaccharides produced from lactose by enzymatic trans-galactosylation and with DP range from 2 to 7 . AOAC Method 2001.02 employs hot $\left(80^{\circ} \mathrm{C}\right)$ phosphate buffer for the extraction of TGOS and lactose from test samples. The extract is treated with $\beta$-galactosidase to hydrolyze the disaccharides and oligosaccharides to yield glucose and galactose. Free galactose and lactose are determined before and after enzyme hydrolysis, and their concentrations are used to calculate the total TGOS content of the test samples [35].

Polydextrose is a manufactured polysaccharide prepared by acid-catalyzed vacuum thermal polymerization of glucose and sorbitol. The average degree of polymerization is 12 with a range of molecular weight between 162 and 20,000. AOAC Method 2000.11 incorporates hot water extraction and ultrafiltration. The filtrate is treated with a mixture of isoamylase, amyloglucosidase, and fructanase. Polydextrose standards are treated similarly and used to quantify a high-molecular-weight fraction of polydextrose using HPAEC-PAD [36].

\subsubsection{Other methods}

Capillary electrophoresis (CE) is a useful technique with the high resolution, high speed of analysis, and the small amounts of analyte required. The advantages of CE compared to chromatographic methods include the extremely simple operation and the low consumption of samples and buffers. However, the main drawback is the lack of sensitivity when low concentration levels are present. Prebiotics, which are carbohydrates, have high ionization constants ( $\mathrm{pKa}$ values of 12 or higher), and therefore they do not carry electrical charges at neutral $\mathrm{pH}$. In addition, they do not absorb UV light above $200 \mathrm{~nm}$, which makes the analysis of prebiotics by CE more difficult. To resolve both problems different procedures have been developed, such as derivatization with direct detection, using chromophore and fluorescent probe carriers of electrical charges, to facilitate detection via UV-VIS absorbance or laserinduced fluorescence (LIF). Although derivatization methods lead to improve sensitivity and resolution, some disadvantages are observed, such as a different reactivity of derivatizing reagents for analytes, formation of several adducts. Derivatization of reducing monosaccharides in $\mathrm{CE}$ is often performed by reductive amination, between the reducing end and an amino group of the tag reagent, using amino with strong chromophores or fluorophores such as 4 -amino benzoic acid and its ethyl ester, 2-aminobenzoic acid, 4-aminobenzonitrile, 2-aminopyridine, etc. In the case of no derivatization, prebiotics after being separated by $\mathrm{CE}$ can be detected by several detectors such as electrical detector, RI detector, or MS detector [26]. 
Mass spectrometry(MS) was also studied for the quantification of prebiotics. Directinfusion Electrospray ionization (ESI) and Matrix-assisted laser desorption/ionization (MALDI) are the most common ionization sources employed for determining the structure of oligosaccharides. Samples are introduced by direct infusion into the ESI ion source. Different analyzers can be coupled to ESI; quadrupole and ion trap are often utilized. In MALDI, the sample is mixed with a matrix, allowed to crystallize by evaporation of the solvent and, submitted to the laser whose energy is absorbed by the matrix and transferred to the carbohydrate which is ionized. The high sensitivity of MALDI allows the detection of oligosaccharides at picomole levels [37]. MALDI has generally coupled to a time of flight (ToF) analyzer results in high sensitivity because most ions generated by the laser are recorded by the detector. A new method for quantification of fructo-oligosaccharides has been published utilizing MALDI/Fourier transforms ion cyclotron resonance (FT-ICR) mass spectrometry [38]. The method was used to monitor the consumption of fructo-oligosaccharides in bacterial fermentation samples to better understand the role of inulin and FOS as prebiotics. Different comparative studies of prebiotic analyzes by HPAECPAD and MALDI have been carried out. HPAEC-PAD is more sensitive in terms of detection limits than MALDI for the analysis of FOS and allowed the separation of linear and branched oligosaccharides. However, MALDI was a faster method and more tolerant to impurities [39].

These methods have good sensitivity and can be performed quickly and conveniently, but require expensive equipment and complicated operation. On the other hand, recent studies using MS methods are mainly applied to the assessment of properties of prebiotics such as the degree of polymerization of prebiotic materials. The fact that different prebiotics can provide fragments with the same $\mathrm{m} / \mathrm{z}$ value will lead to difficulty in characterizing the prebiotics present in the matrix. Therefore, the application of the above methods for the direct quantification of prebiotics in products requires further investigation and improvement in sample preparations.

\subsection{The disadvantages of current analytical methods}

In general, the methods of analysis of prebiotics are currently studied based on different analytical techniques and many methods have been approved by AOAC and AACC. However, the application of these methods in the analysis of food products containing faces many difficulties. The specificity of the method in the analysis of products containing mixed prebiotics is a problem. With the general principle of using enzymes to hydrolyze prebiotics in polymer form to monomers and then, quantification is based on the determination of monomers content. The presence of free monomers (usually monosaccharides) in the matrix leads to positive errors, thus requiring simultaneous analysis of both hydrolyzed and non-hydrolyzed samples, resulting in complex and time-consuming analytical procedures.

On the other hand, the fact that an enzyme can have hydrolytic activity against two prebiotics with similar monomers makes it extremely difficult to identify each prebiotics individually, such as a sample containing both Inulin and FOS. In addition, the complexity of the samples also troubles the analysis. For example, in dairy products containing small amounts of TGOS, a large amount of lactose (up to 50\%) in milk is hydrolyzed simultaneously with TGOS by the enzyme lactase, leading to very large errors in the calculation of results. The problem of LOD and LOQ of the method also needs to be discussed. Currently, analytical methods with high quantitative limits are difficult to meet in analyzing products containing only a small amount of prebiotics. The presence of many other compounds also affects the analytical process. 


\section{CONCLUSIONS}

Prebiotics, which have shown many positive effects on human health, especially the improvement in human intestinal microbiota. This review paper has shown that a variety of analytical methods for the identification and determination of prebiotics have been developed and described. The most common analytical methods and techniques used for determination of prebiotics group are spectrometric methods and chromatographic methods. Spectrophotometric methods have several advantages, such as good analytical specificity and they are easier, less expensive compared with the other methods. However, the spectrophotometric methods are less sensitive than other methods. Chromatographic methods, especially HPAEC-PAD are most commonly used for the quantification of prebiotics. Chromatographic methods generally provide a lower limit of quantification. However, the key issue in the use of chromatographic methods is the separation of the monosaccharides in the mixture after hydrolysis of prebiotics. A major problem with current analytical methods is their efficiency in analyzing a mixture of prebiotics present in food samples. In addition, the removal of other compounds which hydrolyzed along with prebiotics in the sample preparation step is also an unresolved problem. Therefore, in the following years, it is very important to develop quantitative analytical methods for prebiotics in food that can provide a useful tool for assessing the quality of food products that contains prebiotics.

\section{REFERENCES}

[1]. G. R. Gibson, and M. B. Roberfroid, "Dietary modulation of the human colonic microbiota: introducing the concept of prebiotics," Journal of Nutrition, vol.125, no.6, pp. 1401-1412, 1995.

[2]. G. Gibson, K. Scott; R. Rastall, K. Tuohy, A. Hotchkiss, A. Dubert-Ferrandon, M. Gareau, E. Murphy, D. Saulnier, Gunnar Loh, S. Macfarlane, N. Delzenne, Y. Ringel, G. Kozianowski, R. Dickmann, I. Lenoir-Wijnkoop, C. Walker, and R. Buddington, "Dietary prebiotics: current status and new definition," Food Science and Technology Bulletin: Functional Foods, vol. 7, no.1, pp. 1-19, 2010.

[3]. Y. K. Lee and S. Salminen, Handbook of probiotics and prebiotics, 2009.

[4]. J. F. Howlett, V. A. Betteridge, M. Champ, S. A.S. Craig, A. Meheust, and J. M. Jones, “The definition of dietary fiber - discussions at the Ninth Vahouny Fiber Symposium: building scientific agreement," Food \& Nutrition Reseach, vol. 54, 2010.

[5]. K. Younis, S. Ahmad, and K Jahan, "Health benefits and application of prebiotics in foods," Journal of Food Processing \& Technology, vol.6, no.4, pp. 1-7, 2015.

[6]. P. Louis, H. J. Flint, and C. Michel, "How to Manipulate the Microbiota: Prebiotics," Advance in Experimental Medecine and Biology, vol. 902, pp. 119-142, 2016.

[7]. G. T. Macfarlane, H. Steed, and S. Macfarlane, "Bacterial metabolism and health-related effects of galacto-oligosaccharides and other prebiotics," Journal of Applied Microbiology, vol.104, no.2, pp. 305-344, 2008.

[8]. M. M. Ramiro do Carmo, J. C. L. Walker, D. Novello, V. M. Caselato, V. C. Sgarbieri, A. C. Ouwehand, N. A. Andreollo, P. A. Hiane, and E. Freitas Dos Santos, "Polydextrose: Physiological function, and effects on health," Nutrients, vol. 8, no.9, pp. 553, 2016.

[9]. B. A. Stone, "Chemistry of $\beta$-glucans", Chemistry, Biochemistry, and Biology of 1-3 Beta Glucans and Related Polysaccharides, Elsevier, pp. 5-46, 2009.

[10]. J. Knol, G. Boehm, M. Lidestri, F. Negretti, J. Jelinek, M. Agosti, B. Stahl, A. Marini, 
and Fabio Mosca, "Increase of faecal bifidobacteria due to dietary oligosaccharides induces a reduction of clinically relevant pathogen germs in the faeces of formula-fed preterm infants," Acta Paediatrica Supplement, vol. 94, no. 449, pp. 31-33, 2005.

[11]. A. L. McCartney and G. R. Gibson, "The normal microbiota of the human gastrointestinal tract: history of analysis, succession, and dietary influences," Gastrointestinal Microbiology, pp. 51-73, 2006.

[12]. D. B. Silk, A. Davis, J. Vulevic, G. Tzortzis, and G. R. Gibson, "Clinical trial: the effects of a trans-galactooligosaccharide prebiotic on faecal microbiota and symptoms in irritable bowel syndrome," Alimentary Pharmacology \& Therapeutics, vol. 29, no. 5, pp. 508-518, 2009.

[13]. J. O. Lindsay, K. Whelan, A. J. Stagg, P. Gobin, H. O. Al-Hassi, N. Rayment, M. A. Kamm, S. C. Knight, and A Forbes, "Clinical, microbiological, and immunological effects of fructooligosaccharide in patients with Crohn's disease," Gut, vol. 55, no. 3, pp. 348-355, 2006.

[14]. A. Böhm, I. Kaiser, A. Trebstein, and T. Henle, "Heat-induced degradation of inulin," European Food Research and Technology, vol. 220, no. 5, pp. 466-471, 2005.

[15]. S. M. Donovan, "Role of human milk components in gastrointestinal development: current knowledge and future needs," The Journal of Pediatrics, vol. 149, no. 5, pp. S49-S61, 2006.

[16]. G. Veereman-Wauters, S. Staelens, H. Van de Broek, K. Plaskie, F. Wesling, L. C. Roger, A. L. McCartney, and P. Assam, "Physiological and bifidogenic effects of prebiotic supplements in infant formulae", Journal of Pediatric Gastroenterology Nutrition, vol. 52, no. 6, pp. 763 $771,2011$.

[17]. G. Boehm, M. Lidestri, P. Casetta, J. Jelinek, F. Negretti, B. Stahl, and A Marini, "Supplementation of a bovine milk formula with an oligosaccharide mixture increases counts of faecal bifidobacteria in preterm infants," Archives of Disease in Childhood. Fetal and Neonatal Edition, vol. 86, no. 3, pp. F178-181, 2002.

[18]. P. A. M. J. Scholtens, M. S. Alles, J. G. Bindels, E. G. M van der Linde, J. J. M. Tolboom, and J.Knol, "Bifidogenic effects of solid weaning foods with added prebiotic oligosaccharides: a randomised controlled clinical trial," Journal of Pediatric Gastroenterology Nutriton, vol.42, no.5, pp. 553-559, 2006.

[19]. B. V. McCleary, A. Murphy, and D. C. Mugford, "Measurement of total fructan in foods by enzymatic/spectrophotometric method: collaborative study," Journal of AOAC International, vol. 83, no. 2, pp. 356-364, 2000.

[20]. B. V. McCleary and R. Codd, "Measurement of $(1 \rightarrow 3),(1 \rightarrow 4)-\beta$-D-glucan in barley and oats: A streamlined enzymic procedure," Journal of the Science of Food and Agriculture, 55(2), pp. 303-312, 1991.

[21]. B. V. McCleary and D. A. Monaghan, "Measurement of resistant starch," Journal of AOAC International, vol. 85, no. 3, pp. 665-675, 2002.

[22]. H. Ding, C. Li, P. Jin, L. Yuan, Y. Yao, Y. Chen, and Pei Li, "Simultaneous determination of monosaccharides, disaccharides, oligosaccharides and sugar alcohols in foods by high

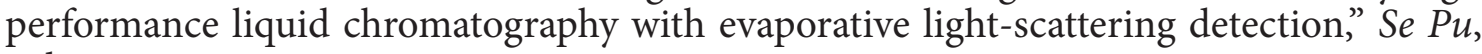
vol. 31, no. 8, pp. 804-808, 2013.

[23]. R. Rodriguez-Gomez, I. Jimenez-Diaz, A. Zafra-Gomez, and J. C. Morales, "Improved sample treatment for the determination of fructooligosaccharides in milk related products by liquid chromatography with electrochemical and refractive index detection", Talanta, vol. 144, pp. 883-889, 2015.

[24]. J. Li, D. Hu, W. Zong, G. Lv, J. Zhao, and S. Li, "Determination of inulin-type fructooligosaccharides in edible plants by high-performance liquid chromatography with 
charged aerosol detector", Journal of Agriculture and Food Chemistry, vol. 62, no. 31, pp. 7707-7713, 2014.

[25]. J. Li, X. Liu, B. Zhou, J. Zhao, and S. Li, "Determination of fructooligosaccharides in burdock using HPLC and microwave-assisted extraction", Journal of Agriculture and Food Chemistry, vol. 61, no. 24, pp. 5888-5892, 2013.

[26]. M. L. Sanz, AI. Ruiz-Matute, N. Corzo, and I. Martínez-Castro, "Analysis of prebiotic oligosaccharides," Prebiotics and Probiotics Science and Technology, pp. 465-534, 2009.

[27]. T. Ikegami, K. Tomomatsu, H. Takubo, K. Horie, and N. Tanaka, "Separation efficiencies in hydrophilic interaction chromatography," Journal of chromatography A, vol. 1184, no. 1-2, pp. 474-503, 2008.

[28]. R. D. Rocklin and C. A. Pohl, "Determination of carbohydrates by anion exchange chromatography with pulsed amperometric detection," Journal of Liquid Chromatography, vol. 6, no. 9, pp. 1577-1590, 1983.

[29]. M. J. Davies and E. F. Hounsell, "Carbohydrate chromatography: towards yoctomole sensitivity," Biomedical Chromatography, vol. 10, no. 6, pp. 285-289, 1996.

[30]. M. Gohlke and V. Blanchard, "Separation of N-glycans by HPLC" Methods in Molecular Biology, vol. 446, pp. 239-254, 2008.

[31]. S. Hase, "Pre-and post-column detection-oriented derivatization techniques in HPLC of carbohydrates," Journal of Chromatography Library, Elsevier, pp. 555-575,1995.

[32]. B. Herbreteau, "Review and state of sugar analysis by high performance liquid chromatography," Analusis (Imprimé), vol. 20, no. 7, pp. 355-374, 1992.

[33]. H. Hoebregs and Collaborators: Balis P De Vries J Eekelen J v Farnell P Gray K Goedhuys B Hermans M Heroff J van Leeuwen M Li BW Martin D Pieters M Quemener B Roomans, "Fructans in foods and food products, ion-exchange chromatographic method: collaborative study," Journal of AOAC International, vol. 80, no. 5, pp. 1029-1039, 1997.

[34]. V. Spichtig, S. Austin, K. Brunt, J. Van Soest, and P. Sanders, "Determination of Fructans in Infant Formula and Adult/Pediatric Nutritional Formula by Anion-Exchange Chromatography with Pulsed Amperometric Detection after Enzymatic Treatment: Collaborative Study, Final Action 2016.14," Journal of AOAC International, vol. 103, no. 5, pp. 1301-1317, 2020.

[35]. J. D. Slegte, "Determination of trans-galactooligosaccharides in selected food products by ion-exchange chromatography: collaborative study," Journal of AOAC International, vol. 85, no. 2, pp. 417-423, 2002.

[36]. S. A. S. Craig, J. F. Holden, and M. Y. Khaled, "Determination of polydextrose in foods by ion chromatography: collaborative study," Journal of AOAC International, vol. 84, no. 2, pp. 472-478, 2001.

[37]. M. Wuhrer, "Glycomics using mass spectrometry," Glycoconjugate journal, vol. 30, no. 1, pp. 11-22, 2013.

[38]. R. R. Seipert, M. Barboza, M. R. Niñonuevo, R. G. LoCascio, D. A. Mills, S. L. Freeman, J. Bruce German, and Carlito B Lebrilla, "Analysis and quantitation of fructooligosaccharides using matrix-assisted laser desorption/ionization Fourier transform ion cyclotron resonance mass spectrometry," Analytical chemistry, vol. 80, no.1, pp. 159-165, 2008.

[39]. J. Wang, P. Sporns, and N. H. Low, "Analysis of food oligosaccharides using MALDI-[34]. J. Wang, P. Sporns, and N. H. Low, "Analysis of food oligosaccharides using MALDI-MS: quantification of fructooligosaccharides," Journal of Agriculture and Food Chemistry, vol. 47, no. 4, pp. 1549-1557, 1999. 


\title{
Tổng quan các phương pháp xác định hàm lượng prebiotic trong thực phẩm
}

\author{
Trần Hùng Sơn ${ }^{1,2^{*}}$, Vũ Thị Thanh An ${ }^{1}$, Mạc Thị Thanh Hoa ${ }^{1}$ \\ Cao Công Khánh ${ }^{1}$, Lê Thị Hồng Hảo ${ }^{1}$ \\ ${ }^{1}$ Viện Kiêm nghiệm an toàn vệ sinh thực phẩm quốc gia, Hà Nội, Việt Nam \\ ${ }^{2}$ Trung tâm Nghiên cứu Thông tin Sản phâm tự nhiên, Viện Khoa học và Công nghệ Hàn Quốc, \\ Gangwon, Republic of Korea
}

Tóm tắt

Prebiotic là một nhóm các chất có khả năng cải thiện hệ thống vi sinh vật đường ruột của con người. Sự tác động của các prebiotic đối với sức khỏe của con người là một lĩnh vực ngày càng được quan tâm và nghiên cứu sâu trong những năm gân đây. Fructo-oligosaccharides và galacto-oligosaccharides là hai loại prebiotic chính được chứng minh có nhiêu tác động có lợi đối với sức khỏe của con người. Bên cạnh đó, ngày càng có nhiều hơn các phân tử carbohydrate cho thấy tiềm năng của một prebiotic. Bài báo này tổng quan sự phân loại, đặc điểm và các phương pháp phân tích prebiotic có trong thực phẩm. Phânn lớn các phương pháp hiện nay được sử dụng là phương pháp sắc ký, đặc biệt là HPAEC-PAD và đông thời còn có các phương pháp hữu ích khác như phương pháp quang phổ. Bài báo cũng chỉ ra nhũ̃ng vấn đề đang tồn tại trong các phương pháp phân tích hiện nay và nhấn mạnh sự cần thiết trong việc phát triển các phương pháp phân tích để định lượng prebiotic trong các nền mẫu thực phẩm phức tạp.

Tư khóa: prebiotics, tông quan, thực phẩm, phương pháp phân tích, fructo-oligosaccharides, galacto-oligosaccharides. 